\title{
Automated calculation of QED corrections to lepton
}

$g-2$

\section{Tatsumi Aoyama*}

Institute of Particle and Nuclear Studies, High Energy Accelerator Research Organization (KEK)

E-mail: aoymepost.kek.jp

\section{Masashi Hayakawa}

Department of Physics, Nagoya University

\section{Toichiro Kinoshita}

Laboratory for Elementary-Particle Physics, Cornell University

\section{Makiko Nio}

Theoretical Physics Laboratory, Nishina Center, RIKEN

\begin{abstract}
This article reports our project on the automated calculation of QED corrections to the anomalous magnetic moment of leptons. Our major concern is the tenth-order correction, which is urgently needed considering the recent improvement of electron $g-2$ measurements. We focus on a type of diagrams that have no internal lepton loops, and have devised the automated code-generating system for the UV-renormalized amplitude. We have newly developed and implemented an efficient algorithm to perform subtractions of IR divergences. This enables us to obtain finite amplitudes that are free from both UV and IR divergences. Currently the numerical evaluation of these diagrams of tenth order is in progress.
\end{abstract}

8th International Symposium on Radiative Corrections

October 1-5, 2007

Florence, Italy

* Speaker. 


\section{Introduction}

The anomalous magnetic moment $g-2$ of the electron is one of the most precisely studied quantities in the particle physics, and it has provided the most stringent test of QED. Recently, a new measurement was carried out by a Harvard group using the Penning trap with cylindrical cavity. Their value announced in 2006 is [1] ${ }^{1}$

$$
a_{e}=1159652180.85(76) \times 10^{-12} \quad[0.76 \mathrm{ppb}],
$$

where $a_{e} \equiv(g-2) / 2$ and the numeral in the parenthesis is the uncertainty in the last two digits of the value. It has a 5.5 times smaller uncertainty than the best previous value [2].

Theoretically, the electron $g-2$ is explained almost entirely by the electromagnetic interaction between electron and photon alone (referred as mass-independent contribution $A_{1}$ ), and it is given as a function of the fine structure constant $\alpha$. The QED correction is evaluated by the perturbation theory as a series in terms of $\alpha$. To match the precision of the recent measurement, the theory must include up to eighth-order corrections. Considering the further improvement of the measurement, it is urgently required to evaluate the actual value of the unknown tenth-order term $A_{1}^{(10)}$. We employ the numerical integration approach, which is the only practical means at present for the eighth and higher order corrections.

The contribution to $A_{1}^{(10)}$ comes from 12672 vertex-type Feynman diagrams, which are classified into 32 gauge-invariant groups within 6 super sets (Set I-VI) according to their structure (Figure 1). 17 groups among these 32 groups have already been evaluated [3]. A particularly difficult one is Set V consisting of 6354 vertex-type diagrams that have no closed lepton loops (called q-type). The difficulty stems from the fact that they are large in number and many of them have very complicated divergence structure. We will present our solution to this obstacle by an automated scheme for code-generation, which enables us to create swiftly the numerical integration codes of the renormalized and finite amplitude of the q-type diagrams $[4,5]$.

\section{Parametric Integration Formalism}

The anomalous magnetic moment $a_{e}$ is given by the static limit of the magnetic form factor. In our formulation, we first employ a relation derived from the Ward-Takahashi identity

$$
\Lambda^{v}(p, q) \simeq-q^{\mu}\left[\frac{\partial \Lambda_{\mu}(p, q)}{\partial q_{v}}\right]_{q \rightarrow 0}-\frac{\partial \Sigma(p)}{\partial p_{v}}
$$

between the self-energy part $\Sigma(p)$ and the sum of the vertex part $\Lambda^{v}(p, q)$. This reduces the number of independent integrals substantially. For the tenth-order Set $\mathrm{V}$ diagrams, the number is, after taking account of the time-reversal symmetry, reduced to 389.

\subsection{Amplitude}

The contribution of a $2 n$ th-order diagram $\mathscr{G}$ is given by an integral over loop momenta of a product of vertices and propagators of leptons and photons. It is turned into a parametric integral

\footnotetext{
${ }^{1}$ Note added: the Harvard group reported a new measurement [16] in which the precision of the value of $a_{e}$ is improved by a factor 2.7 over the value (1.1).
} 


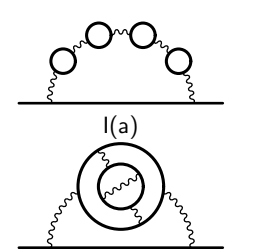

I(g)
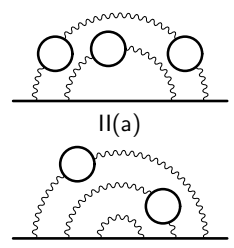

III(a)

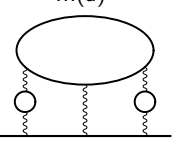

$\mathrm{VI}(\mathrm{a})$

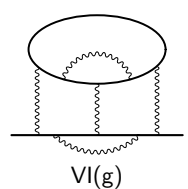

$\mathrm{VI}(\mathrm{g})$

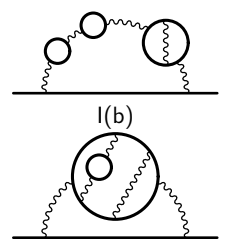

I(h)
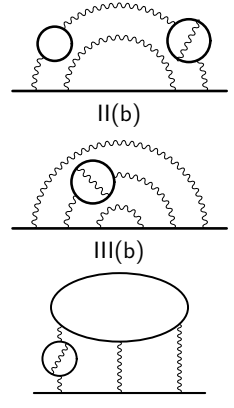

$\mathrm{VI}(\mathrm{b})$

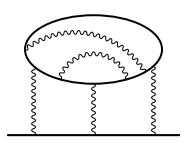

$\mathrm{VI}(\mathrm{h})$
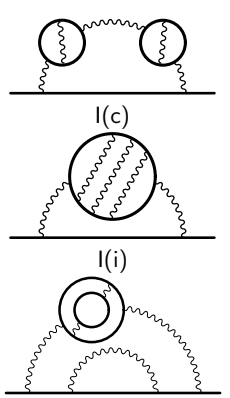

II(c)
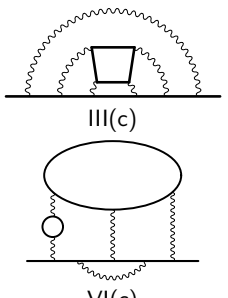

$\mathrm{VI}(\mathrm{c})$

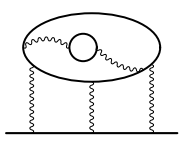

VI(i)
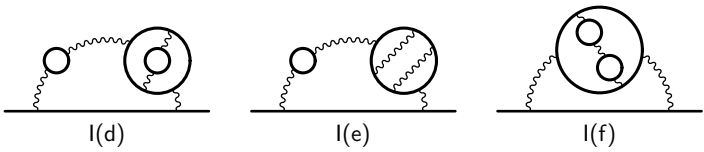

I(f)
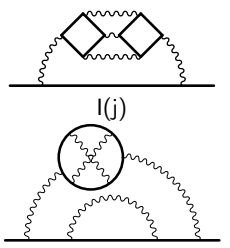

II(d)
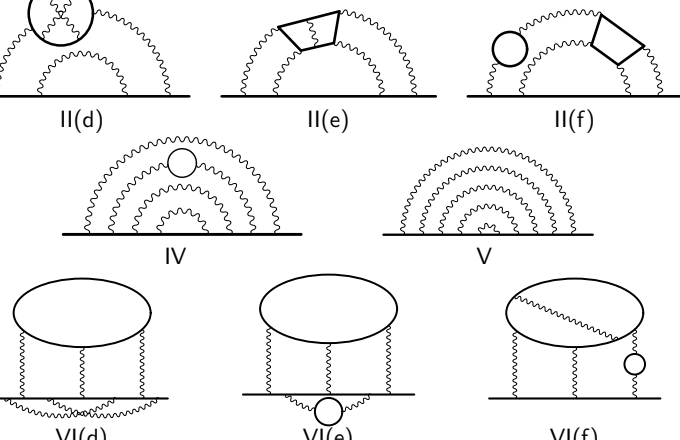

$\mathrm{VI}(\mathrm{f})$

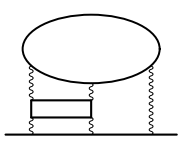

VI(j)

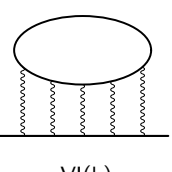

$\mathrm{VI}(\mathrm{k})$

Figure 1: Classification of diagrams contributing to tenth-order QED corrections.

over Feynman parameters $z_{i}$ assigned to the propagators. Carrying out the momentum integration analytically, we can express the amplitude in a concise form

$$
\begin{aligned}
\left.M_{\mathscr{G}}^{(2 n)}=\left(\frac{-1}{4}\right)^{n}(n-1) ! \int(d z)\right)_{\mathscr{G}}\left[\frac { 1 } { n - 1 } \left(\frac{E_{0}+C_{0}}{U^{2} V^{n-1}}+\right.\right. & \left.\frac{E_{1}+C_{1}}{U^{3} V^{n-2}}+\cdots\right) \\
& \left.+\left(\frac{N_{0}+Z_{0}}{U^{2} V^{n}}+\frac{N_{1}+Z_{1}}{U^{3} V^{n-1}}+\cdots\right)\right] .
\end{aligned}
$$

The result is given symbolically as a function of quantities called building blocks, $B_{i j}, A_{j}, U, V$, and $C_{i j}$, which are polynomials of Feynman parameters.

\subsection{Subtraction of UV divergence}

The amplitude constructed above is divergent in general. We adopt subtractive renormalization with the strategy that these divergences are subtracted away by appropriate integrals which cancel the singularities point-by-point on the Feynman parameter space. This scheme is called $K$-operation [6]. The subtraction integral factorizes into an exact lower-order amplitude and the leading UV-divergent part $L_{\mathscr{S}}^{\mathrm{UV}}$ of a vertex-renormalization constant when the divergent subdiagram $\mathscr{S}$ is of vertex type, and into the form $\delta m_{\mathscr{S}}^{\mathrm{UV}} M_{\mathscr{G} / \mathscr{S}}+B_{\mathscr{S}}^{\mathrm{UV}} M_{\mathscr{G} /[\mathscr{S}, j]}$ when $\mathscr{S}$ is of self-energy type. Here, $\delta m_{\mathscr{S}}^{\mathrm{UV}}$ and $B_{\mathscr{S}}^{\mathrm{UV}}$ are the leading UV-divergent parts of mass and wave-function renormalization constants, respectively. When there is more than one divergent subdiagram, the whole UV divergences are identified by the Zimmermann's forests. The subtraction term associated with a forest is obtained by successive operations of $K$-operations of subdiagrams in the forest. 


\subsection{Subtraction of IR divergence}

A diagram suffers from IR divergences when it contains self-energy subdiagrams. In the old approach employed in the numerical evaluation of sixth and eighth orders, the subtraction terms are identified by power-counting rules in a particular limit of Feynman parameters, called I-operation [7]. However, some diagrams have linear divergences that were dealt in an ad hoc way. To this problem, we developed new subtraction schemes called $I$-subtraction and $R$-subtraction. The IR subtraction terms can be identified by the combination of these two subtraction operations. This scheme relies totally on the diagrammatic property and is feasible for the automated treatment.

One type of IR divergence stems from our particular treatment of UV divergence. The $K$ operation for the self-energy subdiagram subtracts away only the leading part $\delta m_{\mathscr{S}}^{\mathrm{UV}}$ of the selfmass term, and the remaining part $\widetilde{\delta m_{\mathscr{S}}} \equiv \delta m_{\mathscr{S}}-\delta m_{\mathscr{S}}^{\mathrm{UV}}$ is regarded to introduce a spurious twopoint vertex effectively. This causes linear or more severe IR divergence. A remedy to this problem is to subtract away the contribution of $\widetilde{\delta m_{\mathscr{S}}}$ by constructing an appropriate integral. We call this operation as $R$-subtraction.

The other type of divergence arises in such a way that when the momenta of photons in the residual diagram $\mathscr{G} / \mathscr{S}$ go to zero the amplitude behaves factorized into a product of the magnetic moment part $M_{\mathscr{S}}$ of the subdiagram $\mathscr{S}$ and the vertex part $\widetilde{L}_{\mathscr{G} / \mathscr{S}(k)}$ in this IR limit. The latter accounts for the logarithmic IR divergence. Thus we prepare a subtraction integral that cancels it. We call this operation as $I$-subtraction.

For more complicated cases involving more than one self-energy subdiagram, the IR subtraction terms are given by the combinations of $I$ - and/or $R$-subtractions. They are recognized by a forest-like structure of self-energy subdiagrams to which the information of distinct types of subtractions is assigned. We call them annotated forests. The whole IR subtraction terms can be identified by finding all possible annotated forests.

\subsection{Residual renormalization}

Thus far we have constructed the renormalized and finite integral of the amplitude of a diagram, but it is not fully equivalent to the standard on-shell renormalization. To complete the calculation, the difference between the full renormalization and the intermediate renormalization achieved so far must be evaluated by collecting all contributions. This step is called the residual renormalization [8].

\section{Automated Calculation}

We developed an automated system that generates numerical integration codes for evaluating q-type diagrams following the steps described in the previous section. It takes a single-line information specifying the form of a diagram as an input, constructs the amplitude and the subtraction terms, and produces FORTRAN codes readily integrated by the numerical integration routine. The system is implemented as a set of Perl programs, and the symbolic manipulations required in the intermediate steps are performed with the helps of FORM [9] and Maple. The numerical integrations are processed by an adaptive Monte Carlo integration routine, VEGAS [10]. Our implementation is applicable to arbitrary order of q-type diagrams. 
For the purpose of testing our system, we applied it to the evaluation of known diagrams of fourth, sixth, and eighth orders. The fourth- and sixth-order contributions obtained by our codes are, after taking account of the residual renormalization, -0.343 95 (53), 0.90526 (53), respectively [5], which reproduce well the exact results of the fourth-order term $-0.344166 \cdots[11,12]$, and the sixth-order term $0.904979 \ldots$ [13], respectively.

For the eighth-order case, we have unexpectedly revealed an inconsistency in the treatment of IR divergences in the previous calculation. With this error corrected, the old calculation, -2.17916 (343), and the new calculation, -2.219 (53), agree within the numerical precision employed [5].

These tests confirmed the validity of our automated system and encourages us to proceed to the evaluation of the tenth-order contribution with confidence. The numerical calculation codes of all 389 independent integrals that represent 6354 diagrams of Set $\mathrm{V}$ have already been obtained by our system, and the numerical evaluation is now in progress.

\section{Concluding Remarks}

In this article we reported our project on the automated calculation of QED corrections to the lepton $g-2$. We developed a new treatment of IR divergences that is suited for automation. We implemented the code-generating system for the type of diagrams that have no closed lepton loops.

The coefficient of the eighth-order contribution is revised to the new value [14]

$$
A_{1}^{(8)}=-1.9144(35)
$$

and it is firmly established by the two independent, mutually consistent evaluations. It should be noted that this is the first independent check of the whole eighth-order term. The value of the fine structure constant determined from the measurement (1.1) and the theory is revised to [15]

$$
\alpha^{-1}\left(a_{e}\right)=137.035999070(98) \text {. }
$$

Our code-generating system is primarily designed for the q-type diagrams, but it can be extended with slight modifications to the types of diagrams obtained by inserting vacuum polarizations to q-type diagrams. The numerical evaluations of Sets III(a), III(b) and IV (see Figure 1) have been carried out. The results will be reported elsewhere.

\section{References}

[1] B. Odom, D. Hanneke, B. D'Urso, and G. Gabrielse, Phys. Rev. Lett. 97 (2006) 030801.

[2] R. S. Van Dyck, P. B. Schwinberg, and H. G. Dehmelt, Phys. Rev. Lett. 59 (1987) 26-29.

[3] T. Kinoshita and M. Nio, Phys. Rev. D 73 (2006) 053007.

[4] T. Aoyama, M. Hayakawa, T. Kinoshita, and M. Nio, Nucl. Phys. B 740 (2006) 138-180.

[5] T. Aoyama, M. Hayakawa, T. Kinoshita, and M. Nio, arXiv:0709.1568 [hep-ph].

[6] P. Cvitanović and T. Kinoshita, Phys. Rev. D 10 (1974) 3978.

[7] P. Cvitanović and T. Kinoshita, Phys. Rev. D 10 (1974) 3991. 
[8] T. Kinoshita in Quantum electrodynamics (T. Kinoshita, ed.), pp. 218-321. World Scientific, Singapore, 1990. (Advanced series on directions in high energy physics, 7).

[9] J. A. M. Vermaseren, math-ph/0010025.

[10] G. P. Lepage, J. Comput. Phys. 27 (1978) 192.

[11] A. Petermann, Helv. Phys. Acta 30 (1957) 407-408.

[12] C. M. Sommerfield, Phys. Rev. 107 (1957) 328-329.

[13] S. Laporta and E. Remiddi, Phys. Lett. B379 (1996) 283-291.

[14] T. Aoyama, M. Hayakawa, T. Kinoshita, and M. Nio, Phys. Rev. Lett. 99 (2007) 110406.

[15] G. Gabrielse, D. Hanneke, T. Kinoshita, M. Nio, and B. Odom, Phys. Rev. Lett. 98 (2007) 039902.

[16] D. Hanneke, S. Fogwell, and G. Gabrielse, arXiv:0801.1134 [physics.atom-ph]. 